\title{
Mach-Zehnder Interferometer Polarization Splitter in InGaAsP/InP
}

\author{
L. B. Soldano, A. H. de Vreede, M. K. Smit, B. H. Verbeek, E. G. Metaal and F. H. Groen
}

\begin{abstract}
A passive TE/TM mode polarization splitter based on a Mach-Zehnder interferometer is demonstrated. Insertion loss of $1.5 \mathrm{~dB}$ and extinction ratios of $-19 \mathrm{~dB}$ for TE and -15 $\mathrm{dB}$ for TM have been measured at 1510-nm wavelength. The device attains large optical bandwidth employing a pair of multimode interference (MMI) couplers and a wavelength-tolerant birefringent structure.
\end{abstract}

\section{INTRODUCTION}

$\mathbf{S}$ PATIAL TE/TM mode splitting is crucial for polarization diversity receivers [1], polarization shift keying [2] and polarization diversity multiplexing [3]. Several polarization splitters have been studied based on different operating principles. Asymmetric $Y$-branches on $\mathrm{LiNbO}_{3}$ were reported [4] in which the effective indices were electro-optically controlled. The polarization-dependent phase shift in a pair of length-compensated nitride-loaded silicon-based waveguides was exploited in an interferometer polarization splitter [5]. Polarization splitters based on metal-cladded directional couplers in InGaAsP/InP were demonstrated [6]. More recently, a mode evolution splitter has been reported [7] which exploits the large birefringence of first-order modes in ridge waveguides. Here we report the design, fabrication and measurement results of a passive Mach-Zehnder Interferometer (MZI) polarization splitter in InGaAsP/InP. We demonstrate that waveguides with equal TE propagation constants - while still having a convenient difference in TM - can be realized by suitably loading a ridge waveguide with a dielectric $\left(\mathrm{SiO}_{2}\right)$ layer and a metal layer on top. Based on this concept, two equallength waveguides (which we call differential arms) can be fabricated capable of providing the desired TM phase shift of $\pi$ within the MZI, without introducing any TE phase shift. This approach has the advantage of avoiding the need of a critical compensating length in the differential arms, and/or the tuning of the waveguide widths.

\section{PRINCIPLE OF OPERATION}

A schematic layout of the Mach-Zehnder interferometer polarization splitter is shown in Fig. 1. The device consists

L. B. Soldano, A. H. de Vreede, M. K. Smit, and B. H. Verbeek are with Delft University of Technology, Faculty of Electrical Engineering, Laboratory of Telecommunication and Remote Sensing Technology, P.O. Box 5031, 2600 GA Delft, The Netherlands.

E. G. Metaal is with Royal PTT Nederland N.V., PTT Research Leidschendam, The Netherlands.

F. H. Groen is with Delft University of Technology, Faculty of Applied Physics, Research Group for Optics, The Netherlands. IEEE Log Number 9400072 of two 3-dB MMI couplers in restricted resonance [8], and a pair of non-equally birefringent straight waveguides of equal length (differential arms). These arms are designed in such a way as to present no relative phase shift for TE-polarization, and a relative phase shift of $\pi$ for TM-polarization, i.e.

$$
\begin{aligned}
\Delta \varphi_{\mathrm{TE}} & =k_{0}\left(N_{\mathrm{TE}}^{\mathrm{ms}}-N_{\mathrm{TE}}^{\mathrm{sl}}\right) L_{D}=k_{0} \Delta N_{\mathrm{TE}} L_{D}=0 \\
\Delta \varphi_{\mathrm{TM}} & =k_{0}\left(N_{\mathrm{TM}}^{\mathrm{ms}}-N_{\mathrm{TM}}^{s l}\right) L_{D}=k_{0} \Delta N_{\mathrm{TM}} L_{D}=\pi
\end{aligned}
$$

where $k_{0}=2 \pi / \lambda$ is the wavenumber, $\lambda$ is the free-space wavelength, $N$ is the effective refractive index, $L_{D}$ is the length of the differential arms, and the superscripts ms and sl refer to the metal-SiO ${ }_{2}$ cladded waveguide and the strip-loaded waveguide respectively.

The first 3-dB coupler splits the (randomly polarized) input signal into two quadrature $\left(+90^{\circ}\right.$ relative phase) components, which are fed into the differential arms. Because of the equal TE propagation constants, the TE components will keep their relative phase $\left(+90^{\circ}\right)$ all along the differential arms and, when combined in the second 3-dB coupler, will add up and come out at the cross output port. The TM components, due to the phase shift of $\pi$ introduced by one arm, will reach the second 3-dB coupler with a relative phase of $-90^{\circ}$ and, when combined, will come out at the bar output port.

\section{DESIGN CONSIDERATIONS}

A metal layer applied directly on top of a ridge waveguide causes a very strong reduction in the TM effective index [6], with a small decrease in the TE effective index and a large attenuation for both polarizations. The inclusion of a (low-index) dielectric layer between the waveguide and the metal can compensate the TE index decrease and bring the propagation losses to acceptable levels [9], while still providing a useful difference in TM effective index. This allows the design of a metal-SiO${ }_{2}$ cladded waveguide with equal TE effective index as the strip-loaded waveguide. A cross section of the metal-SiO $\mathrm{S}_{2}$ cladded and strip-loaded waveguide structures is shown in Fig. 2.

The real and imaginary part of the effective indices were calculated for both structures by using the Effective Index Method with complex refractive indices for the metal layers [10]. The Fiedler and Schlachetzki model [11] was used to calculate the refractive index of InP and of lattice-matched quaternary $\operatorname{In}_{0.72} \mathrm{Ga}_{0.28} \mathrm{As}_{0.61} \mathrm{P}_{0.39}\left(\lambda_{g}=1.3 \mu \mathrm{m}\right)$ at different wavelengths. Fig. 3 shows the differences in the real part 


\section{TE+TM}

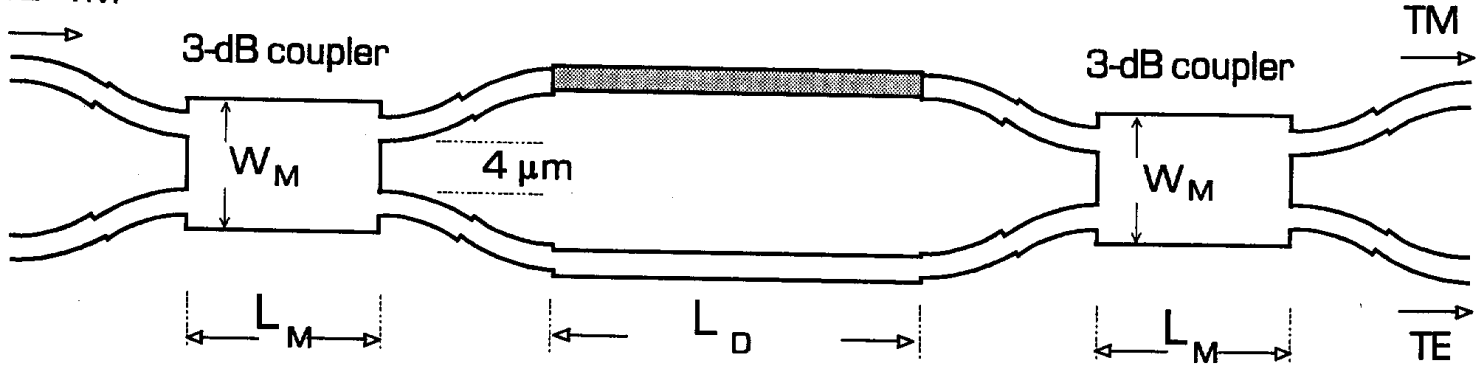

Fig. 1. Schematic layout of the Mach-Zehnder interferometer polarization splitter. It consists of two MMI 3-dB couplers with length $L_{M}$ and width $W_{M}$, and a pair of differential arms of length $L_{D}$. The shaded area indicates the metal-SiO $\mathrm{O}_{2}$ cladding on top of the strip-loaded waveguide.

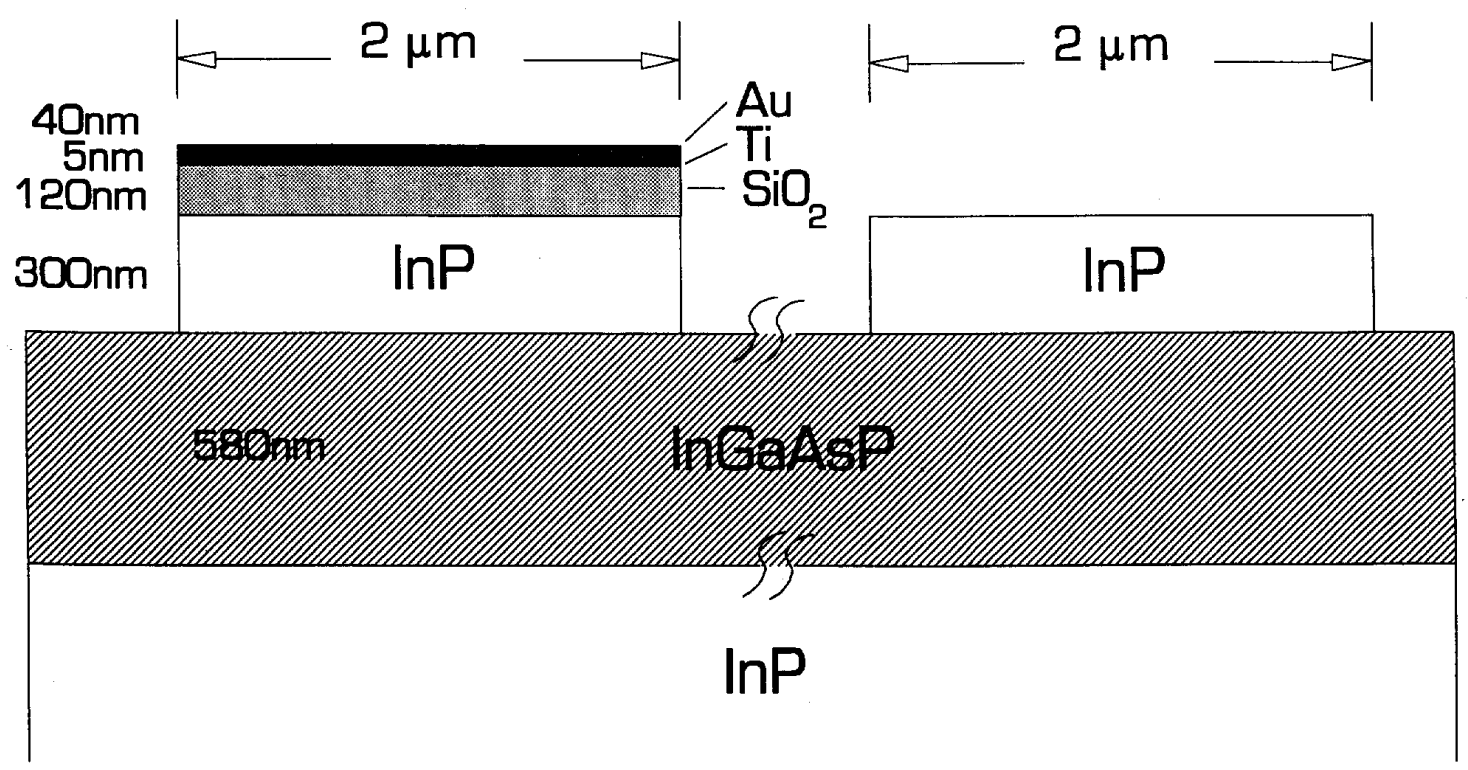

Fig. 2. Cross section of the metal- $\mathrm{SiO}_{2}$ cladded waveguide (left) and the strip-loaded waveguide (right) structures. The refractive indices are: $n_{\mathrm{Au}}=0.18-j 10.2, n_{\mathrm{Ti}}=3.70-j 4.50[10], n_{\mathrm{InP}}=3.17, n_{\mathrm{InGaAsP}}=3.39[11]$, and $n_{\mathrm{ox}}=1.444$ [17] at 1550-nm wavelength.

of the effective indices $\Delta N_{\mathrm{TE}}$ and $\Delta N_{\mathrm{TM}}$ (as defined in eqs. (1_ and (2)) between the metal- $\mathrm{SiO}_{2}$ cladded and the strip-loaded waveguide, calculated as a function of the $\mathrm{SiO}_{2}$ layer thickness $d_{\text {ox }}$. Metal layers thicker than $30 \mathrm{~nm}$ do not further modify the effective refractive index differences. At $d_{\mathrm{ox}} \approx 120 \mathrm{~nm}$, we find $\Delta N_{\mathrm{TE}}=0$ which fulfills (1) for any value of $L_{D}$. For this value of $d_{\mathrm{ox}}, \Delta N_{\mathrm{TM}}=4.3 \times 10^{-4}$ and (2) is thus fulfilled with $L_{D}=1.76 \mathrm{~mm}$. The attenuation penalty due to the presence of metal in the metal- $\mathrm{SiO}_{2}$ cladded waveguide decreases exponentially with the $\mathrm{SiO}_{2}$ thickness, and for $d_{\mathrm{ox}}=120 \mathrm{~nm}$ we calculated it to be $\sim 1.8 \mathrm{~dB} / \mathrm{cm}$ for TE and $\sim 0.8 \mathrm{~dB} / \mathrm{cm}$ for TM.

We used MMI couplers because of their good fabrication tolerances, polarization independence and large optical bandwidth [12]. All access waveguides were $2-\mu \mathrm{m}$ wide. In order to minimize losses [13], lateral offsets were applied at the straight-to-curve transitions $(0.15 \mu \mathrm{m})$ and at the curve-tocurve transitions $(0.30 \mu \mathrm{m})$. The total device is $3.3-\mathrm{mm}$ long, which includes $1.0-\mathrm{mm}$ radius curved access waveguides with a $50-\mu \mathrm{m}$ separation.

\section{FABRICATION OF THE DEVICES}

The devices were realized on a non-intentionally doped InP/InGaAsP/InP wafer grown by low-pressure MOVPE [14]. After sputtering a $120-\mathrm{nm} \mathrm{SiO}{ }_{2}$ film onto the whole wafer [17], a thin $(\sim 5 \mathrm{~nm}) \mathrm{Ti}$ layer (to improve the adherence of $\mathrm{Au}$ ) and a 40-nm Au layer were deposited by e-beam evaporation. The entire layout was first defined by a standard photolithography process and then patterned with two reactive-ion etching (RIE) steps. Metals and $\mathrm{SiO}_{2}$ layers were etched in a $\mathrm{CHF}_{3}$ plasma RIE. The $300-\mathrm{nm}$ InP top layer was etched by a self-aligned $\mathrm{CH}_{4}: \mathrm{He} \mathrm{RIE}$ [15]. A second (non-critical alignment) standard photolithography covers one differential arm of the device, allowing the removal of the unwanted metals and $\mathrm{SiO}_{2}$ by 


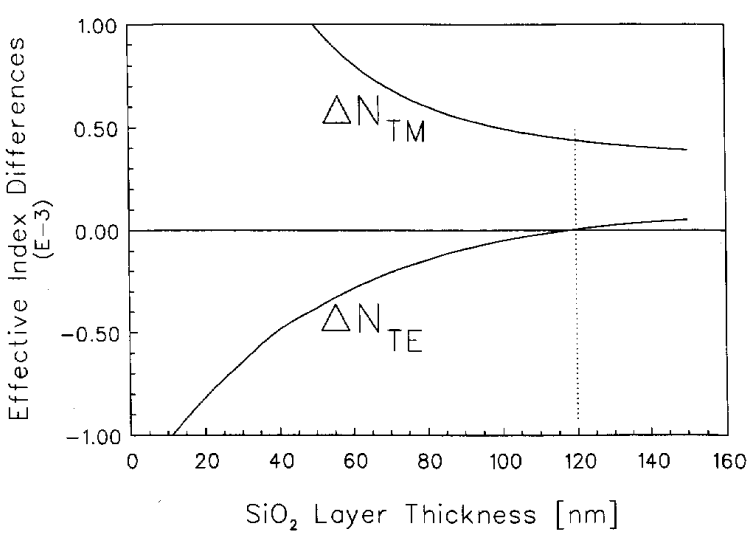

Fig. 3. Calculated refractive index differences $\Delta N_{\mathrm{TE}}$ and $\Delta N_{\mathrm{TM}}$ (from eqs. (1) and (2)) between the metal- $\mathrm{SiO}_{2}$ cladded waveguide and the strip-loaded waveguide, as a function of $\mathrm{SiO}_{2}$ layer thickness $d_{\mathrm{ox}}$. The dotted line indicates the design value of $d_{\mathrm{ox}}$ for which $\Delta N_{\mathrm{TE}}=0$.

wet chemical etching. The samples were anti-reflection coated by depositing a $\lambda / 4$ layer of $\mathrm{SiO}_{x}$ on the cleaved facets.

\section{EXPERIMENTAL RESULTS}

We fabricated a series of MZI polarization splitters with $L_{D}$ varying from 1.5 to $2.0 \mathrm{~mm}$ in steps of $20 \mu \mathrm{m}$ and MMI 3-dB couplers with $L_{M}=425 \mu \mathrm{m}$ and $W_{M}=15.8,16.0$ and $16.2 \mu \mathrm{m}$. In order to test the individual performances of the MMI 3-dB couplers, we integrated a series of them with lengths $L_{M}$ ranging from 415 to $435 \mu \mathrm{m}$ in steps of $5 \mu \mathrm{m}$, and widths $W_{M}$ ranging from 15.6 to $16.4 \mu \mathrm{m}$ in steps of $0.2 \mu \mathrm{m}$. These $3-\mathrm{dB}$ couplers showed excess loss below $1.0 \mathrm{~dB}$ and imbalance within $\pm 0.5 \mathrm{~dB}$, for a length tolerance of $\pm 5 \mu \mathrm{m}$ and a width tolerance of $\pm 0.2 \mu \mathrm{m}$. Attenuation on $2-\mu \mathrm{m}$ wide strip-loaded straight (reference) waveguides was determined to be $1.2 \mathrm{~dB} / \mathrm{cm}$ for TE and $2.8 \mathrm{~dB} / \mathrm{cm}$ for TM, from transmission measurements, a twodimensional overlap calculation of the mode mismatch, and an analytical approximation of the facet reflectivity [16]. These results agree to within $\pm 0.3 \mathrm{~dB} / \mathrm{cm}$ with loss measurements performed on similar waveguide structures by the FabryPerot resonance method. The metal- $\mathrm{SiO}_{2}$ cladded waveguides showed attenuations of $3.3 \mathrm{~dB} / \mathrm{cm}$ for TE and $3.7 \mathrm{~dB} / \mathrm{cm}$ for TM.

The MZI polarization splitters were characterized by launching alternatively TE- and TM-polarized light into one input, recording the light from both outputs and repeating the process for the other input. Light was end-fire coupled by focusing a pair of $40 \times$ IR anti-reflection coated microscope objectives at the cleaved facets. The outputs were imaged onto a $\mathrm{Ge}$ photodiode and read by a lock-in amplifier.

Fig. 4 shows the insertion loss and extinction ratio measured with a 1507-nm Fabry-Perot laser on a number of devices with varying $L_{D}$. Typical insertion loss of $1.5 \mathrm{~dB}$ (with respect to straight reference waveguides) was observed for both polarizations in the vicinity of $L_{D}=1.76 \mathrm{~mm}$. As expected, TE extinction ratio remains quite constant for all devices. Its slight droop is most probably due to the fact that

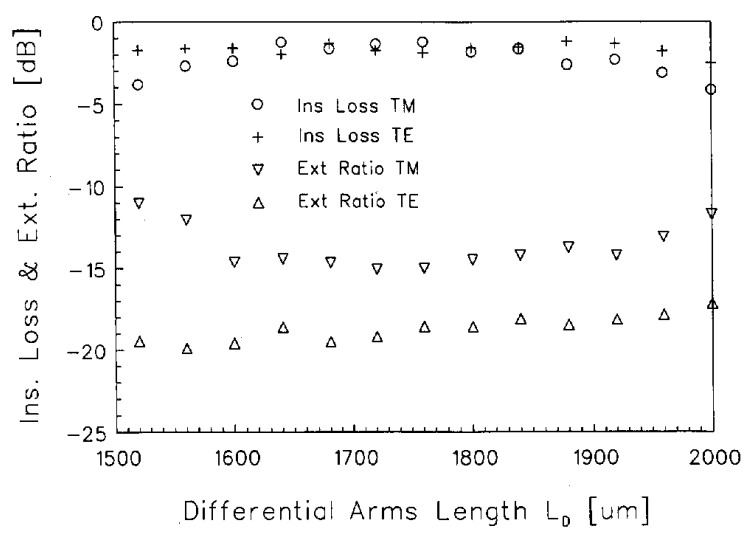

Fig. 4. Measured insertion loss and extinction ratio as a function of differential arms length $L_{D}$, obtained with a $1507-\mathrm{nm}$ centre wavelength FP laser.

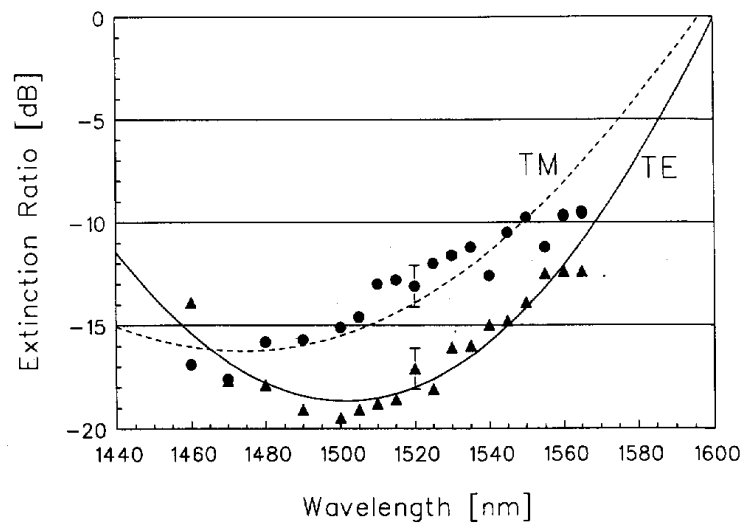

Fig. 5. Measured extinction ratio versus wavelength for a device with $L_{D}=1.76 \mathrm{~mm}$ obtained with an external cavity tunable laser. Each marker is the average of two measurements (one for each input), with the typical observed spread shown as vertical bars. Solid and dashed lines are parabolic fits.

$\Delta N_{\mathrm{TE}}$ (though very low) is not exactly zero. The extinction ratio for TM shows a soft maximum around $L_{D}=1.76 \mathrm{~mm}$. The difference between TE and TM extinction ratios is partly due to a somewhat larger TM imbalance in the MMI 3-dB couplers.

Fig. 5 shows the extinction ratio as a function of the wavelength, measured on a device with design value dimensions $\left(L_{D}=1.76 \mathrm{~mm}, L_{M}=425 \mu \mathrm{m}\right.$ and $\left.W_{M}=16.0 \mu \mathrm{m}\right)$. Each marker represents the average of two measurements (one for each excited input port). The best TE extinction ratio was -19 $\mathrm{dB}$ at $1500 \mathrm{~nm}$, in good agreement with the design wavelength of $1510 \mathrm{~nm}$. TM reaches a best extinction ratio of $-17 \mathrm{~dB}$ at $1470 \mathrm{~nm}$. We attribute this discrepancy to the uncertainty in the value of the metals refractive indices. Over a $60-\mathrm{nm}$ wavelength range the TE (TM) extinction ratio remains below $-16 \mathrm{~dB}(-13 \mathrm{~dB})$.

\section{CONCLUSION}

A polarization splitter based on a Mach-Zehnder interferometer has been demonstrated. The desired TE and TM 
phase shifts were realized with a metal- $\mathrm{SiO}_{2}$ cladding on a strip-loaded waveguide. The devices were fabricated in In$\mathrm{GaAsP} / \mathrm{InP}$ waveguides with a rather simple two-mask process. The design requires, however, a good control of the $\mathrm{SiO}_{2}$ layer thickness and its refractive index, in order to accurately compensate the influence of the metal layer for TE polarization. For example, $\mathrm{a} \pm 10 \%$ deviation from the optimum $\mathrm{SiO}_{2}$ layer thickness would result in a $5-\mathrm{dB}$ penalty in the extinction ratio. At $1510-\mathrm{nm}$, we measured extinction ratios of $-19 \mathrm{~dB}$ for TE and $-15 \mathrm{~dB}$ for TM, insertion losses of about $1.5 \mathrm{~dB}$ for both polarizations, and large bandwidth.

\section{ACKNOWLEDGMENT}

The authors wish to acknowledge fruitful discussions with Y. S. Oei regarding process technology and would like to thank A. Kuntze for depositing the AR coating. The wafers were grown by IMEC, University of Gent, Belgium. This work was partially supported by the Technical Sciences Foundation (STW) within the programme of the Dutch Foundation for Fundamental Research on Matter (FOM).

\section{REFERENCES}

[1] T. Okoshi, S. Ryn and K. Kikuchi, "Polarization diversity receiver for heterodyne/coherent optical fiber communications," in Proc. IOOC/OFC'83, Tokyo, Japan, 1983, paper 30C3-2.

[2] K. Fukuchi, S. Yamazaki, T. Ono and M. Rangaraj, "Polarization shift keying - direct detection scheme for fiber nonlinear effect insensitive communication system," in Proc. European Conference on Optical Communication ECOC'92, Berlin, Germany, 1992, pp. 169-172.

[3] F. Heismann, P. B. Hansen, S. K. Korotky, G. Raybon, J. J. Veselka and M. S. Whalen, "Automatic polarization demultiplexer for polarizationmultiplexed transmission systems," in Proc. European Conference on Optical Communication ECOC'93, Montreux, Switzerland, 1993, pp. $401-404$.
[4] M. Masuda and G. L. Yip, "An optical TE-TM mode splitter using a $\mathrm{LiNbO}_{3}$ branching waveguide," Appl. Phys. Lett., vol. 37, pp. 20-22. 1980.

[5] Y. Shani, C. H. Henry, R. C. Kistler and K. J. Orlowsky, "Four-port integrated optic polarization splitter," Appl. Opt., vol. 29, no. 3, pp. 337-339, 1990.

[6] P. Albrecht, M. Hamacher, H. Heidrich, D. Hoffmann, H.-P. Nolting and C. M. Weinert, "TE/TM mode splitters on InGaAsP/InP," IEEE Photon. Technol. Lett., vol. 2, no. 2, pp. 114-115, 1990.

[7] J. W. Pedersen, J. J. G. M. van der Tol, E. G. Metaal, Y. S. Oei, F. H Groen and I. Moerman, "Realization of a mode evolution polarization splitter on InGaAsP/InP," in Proc. European Conference on Optical Communications ECOC' 93 , Montreux, Switzerland, 1993, post-deadline paper ThC12.3.

[8] L. B. Soldano, F. B. Veerman, M. K. Smit, B. H. Verbeek, A. H. Dubost and E. C. M. Pennings, "Planar monomode optical couplers based on multimode interference effects," J. Lightwave Technol., vol. 10, no. 12, pp. 1843-1850, 1992.

[9] S. C. Rashleigh, "Four-layer metal-clad thin film optical waveguides," Optical and Quantum Electronics, vol. 8, pp. 49-60, 1976.

[10] M. A. Ordal, L. L. Long, R. J. Bell, S. E. Bell, R. R. Bell, R. W Alexander and C. A. Ward, "Optical properties of the metals Al, Co, $\mathrm{Cu}, \mathrm{Au}, \mathrm{Fe}, \mathrm{Pb}, \mathrm{Ni}, \mathrm{Pd}, \mathrm{Pt}, \mathrm{Ag}, \mathrm{Ti}$, and $\mathrm{W}$ in the infrared and near infrared," Appl. Opt., vol. 22, no. 7, pp. 1099-1119, 1983.

[11] F. Fiedler and A. Schlachetzki, "Optical parameters of InP-based waveguides," Solid-State Electron., vol. 30, no. 1, pp. 73-83, 1987.

[12] L. B. Soldano, M. Bachmann, P.-A. Besse, M. K. Smit and H. Melchior, "Large optical bandwidth of InGaAsP/InP multi-mode interference 3-dB couplers," in Proc. European Conference on Integrated Optics ECIO'93, Neuchâtel, Switzerland, 1993, paper 14-10.

[13] E. C. M. Pennings, Bends in Optical Ridge Waveguides, Modeling and Experiments, Ph.D. Thesis, Delft University of Technology, 1990. ISBN 90-9003413-7.

[14] I. Moerman, G. Coudenys, P. Demeester, B. Turner and J. Cawley, "Influence of gas mixing on the lateral uniformity in horizontal MOVPE reactors," J. Cryst. Growth, vol. 107, pp. 175-180, 1991.

[15] L. H. Spiekman, F. P. G. M. van Ham, M. Kroonwijk, Y. S. Oei, J. J. G. M. van der Tol, F. H. Groen and G. Coudenys, "A new fabrication process for very low-loss narrow-width InGaAsP/InP waveguides," in Proc. European Conference on Integrated Optics ECIO'93, Neuchâtel, Switzerland, 1993, paper 2-30.

[16] J. Buus, "Analytical approximation for the reflectivity of DH lasers," IEEE J. Quantum Electron., vol. QE-17, no. 12, pp. 2256-2257, 1981

[17] M. K. Smit, "Integrated Optis in Silicon-based Aluminum Oxide", Ph.D. Thesis, Delft University of Technology, 1991. ISBN 90-9004261-X. 